\title{
Self-injury: Treatment, Assessment, Recovery (STAR): online intervention for adolescent non-suicidal self-injury - study protocol for a randomized controlled trial
}

Michael Kaess ${ }^{1,2^{*}}$, Julian Koenig ${ }^{1,2}$, Stephanie Bauer ${ }^{3}$, Markus Moessner $^{3}$, Gloria Fischer-Waldschmidt ${ }^{4}$, Margarete Mattern ${ }^{5}$, Sabine C. Herpertz ${ }^{5}$, Franz Resch ${ }^{4}$, Rebecca Brown ${ }^{6}$, Tina In-Albon ${ }^{7}$, Michael Koelch ${ }^{8}$, Paul L. Plener ${ }^{6,9}$, Christian Schmahl ${ }^{10}$, Alexandra Edinger ${ }^{2,4}$ and the STAR Consortium

\begin{abstract}
Background: Non-suicidal self-injury (NSSI) is a clinically significant behavior affecting approximately $18 \%$ of adolescents and young adults worldwide. The importance of NSSI is supported by its association with a broad spectrum of mental disorders. Despite its high relevance, evidence-based, specific, time-, and cost-effective treatment approaches are scarce. Cognitive behavioral therapy (CBT) seems effective in reducing the frequency of NSSI in adolescents and young adults. However, young people are often reluctant to seek professional help and effective interventions adressing NSSI are not sufficiently available across all regions of Germany. Research indicates that the majority of youth with risk behavior (including NSSI) prefer technology-based interventions. To date, effective interventions for adolescents and young adults with NSSI that are deliverd online are not available.

Methods: The present project aims to develop and evaluate an online intervention for adolescents and young adults with NSSI based on the content of a recently evaluated face-to-face short-term program that includes elements of CBT and dialectical behavior therapy (DBT): "The Cutting Down Programme" (CDP). The efficacy of the new online CDP intervention will be tested in a randomized controlled trial (RCT) in which $n=700$ youths engaging in repetitive NSSI will participate in either an online psychoeducation $(n=350)$ or online CDP $(n=350)$. Within a postline assessment four months after baseline (end of treatment; T1), and follow-up evaluations 12 and 18 months after baseline (follow-ups; T2 and T3), NSSI and comorbid symptoms as well as quality of life will be assessed. It is hypothesized that participants receiving online CDP report a greater reduction in the frequency of NSSI within the last three months at T2 (primary endpoint) compared to those receiving online psychoeducation. Exploratory analyses will focus on predictors of treatment outcome.
\end{abstract}

Discussion: We report on the development and evaluation of an online intervention for adolescents and young adults engaging in NSSI based on the CDP. If supported by empirical evidence, an online-based intervention for NSSI might help to overcome the limited availability of adequate interventions for youth.

Trial registration: German Clinical Trials Register, DRKS00014623. Registered on 22 May 2018.

Keywords: Non-suicidal self-injury, Adolescents, Internet, Online intervention, Randomized controlled trial

\footnotetext{
* Correspondence: michael.kaess@med.uni-heidelberg.de

'University Hospital of Child and Adolescent Psychiatry and Psychotherapy,

University of Bern, Bern, Switzerland

${ }^{2}$ Section for Translational Psychobiology in Child and Adolescent Psychiatry,

Department of Child and Adolescent Psychiatry, Centre for Psychosocial

Medicine, University Hospital Heidelberg, Heidelberg, Germany

Full list of author information is available at the end of the article
}

(c) The Author(s). 2019 Open Access This article is distributed under the terms of the Creative Commons Attribution 4.0 International License (http://creativecommons.org/licenses/by/4.0/), which permits unrestricted use, distribution, and

reproduction in any medium, provided you give appropriate credit to the original author(s) and the source, provide a link to the Creative Commons license, and indicate if changes were made. The Creative Commons Public Domain Dedication waiver (http://creativecommons.org/publicdomain/zero/1.0/) applies to the data made available in this article, unless otherwise stated. 


\section{Background}

Non-suicidal self-injury (NSSI) affects approximately $17-18 \%$ of adolescents worldwide $[1,2]$. NSSI is characterized by the intentional and self-inflicted destruction of body tissue without suicidal intent [3]. The importance of NSSI is highlighted by recent research illustrating that NSSI is an important predictor of suicidal behavior [4-6]. Furthermore, it is highly associated with other riskbehaviors and comorbid psychopathology [7, 8]. NSSI is a common and highly recurrent behavior that peaks in adolescence [4, 9-11].

It is essential to develop interventions supporting adolescents with NSSI to stop the behavior. However, only few interventions have been developed that specifically target NSSI despite its high clinical pertinence [12-14]. NSSI, particularly in the context of (emerging) borderline personality disorder (BPD), is often treated with Dialectical Behavior Therapy for adolescents (DBT-A), which has shown to be effective [15]. Other treatments that seem effective for reducing NSSI frequency are Mentalization-Based Treatment for adolescents (MBT-A) as well as Cognitive Behavioral Therapy (CBT) [16, 17]. A recent meta-analysis [16] suggested that DBT, MBT, and CBT may all be effective for treating NSSI. However, comprehensive access to these treatments is restricted due to limited resources and a lack of specially trained clinicians [18]. Given the limited resources in the mental healthcare field, it remains a matter of discussion if DBT-A and MBT-A, which are targeted at youth with BPD need to be fully implied.

Thus, less intensive yet effective programs that focus specifically on NSSI are needed to improve the general standard of care for affected individuals [19], particularly for adolescents reluctant to seek professional help despite their actual need. Indeed, help-seeking in adolescents engaging in NSSI is considerably low [20-22]. Adolescents endorsing NSSI express more negative attitudes towards help-seeking compared to past self-injurers and adolescents with no history of NSSI [22]. Negative attitudes towards help-seeking in adolescents and young adults with NSSI frequently arise from the disclosure and related stigma of the behavior towards parents, peers, and mental health professionals [23]. Typically, adolescents engaging in NSSI turn to the Internet in order to find peer support [24]. Although such exchange may actually foster NSSI, by reinforcement through the sharing of stories and strategies of NSSI [25], it also offers opportunities for therapeutic interventions [26]. Studies [27, 28] have shown that a significant percentage of adolescents and young adults (45-93\%) with risk behavior (including NSSI) prefer a technology-based intervention format (versus an in-person face-to-face intervention). Such youth-adequate delivery of a NSSI intervention may lower barriers towards care and reach adolescents and young adults in rural areas, where adequate help is not sufficiently available. To date, the access to effective intervention requires great efforts and waiting times, especially for adolescents and young adults in rural regions. We have previously shown that the time that adolescents need to travel to receive professional help for their mental health problems hinders actual helpseeking behavior [29]. Online-based interventions could overcome these barriers.

However, to date, no effective online-based intervention for adolescents engaging in NSSI exists. Time- and costeffective brief interventions exclusively targeting NSSI are rare. Therefore, we have developed an online intervention for adolescents and young adults with NSSI based on the content and our experience with a cognitive-behavioral short-term program for adolescents engaging in NSSI. In 1999, a short-term psychotherapy was developed for adults exhibiting deliberate self-harm, the manual-assisted cognitive-behavior therapy (MACT) [30]. In 2011, the MACT was adapted for adolescents within "The Cutting Down Programme" (CDP) [31], consisting of 8-12 sessions. Our group has just completed the first randomized controlled trial (RCT) on the effectiveness of face-to-face CDP [19] including $n=74$ adolescents engaging in NSSI. Findings are promising and are currently under review.

To deliver such effective interventions online may increase the availability and offer the opportunity to provide adolescents and young adults concerned with adequate intervention [32]. Concerning the low help-seeking behavior within the particular target group, the low threshold access, low stigma, and high confidentiality offered by online interventions are promising in reducing the treatment gap. Consequently, our proposed online intervention could lower barriers for a high-risk target group to seek professional help.

\section{Objectives and outcomes}

The main objective of the present study is to evaluate a newly developed, easily accessible, online intervention program for adolescents and young adults engaging in NSSI, based on an existing short-term face-to-face manualized intervention (CDP). The effectiveness of online CDP will be evaluated in a RCT in comparison to an online psychoeducation program. The main outcome of the trial is the reduction in the frequency of NSSI within the last three months assessed by the NSSI Severity Questionnaire (NSSV-SG) [33] at one-year follow-up (primary endpoint; T2).

Secondary outcome criteria are health-realted quality of life as well as comorbid psychopathology and suicidal behavior.

\section{Primary hypothesis}

Participants receiving online CDP will show a significantly greater reduction in the frequency of NSSI within the past three months at T2 compared to participants within the online psychoeducation group. 


\section{Methods/Design}

\section{Setting and recruitment}

The present trial is a study within the consortium "Self-injury-Treatment: Assessment, Recovery (STAR)." Cooperating study sites (Ulm, Landau, Mannheim, Karlsruhe, and Berlin) will investigate the natural course of NSSI as well as psychocological and neurobiological predictors and improve ways to dessiminate specific knowledge on NSSI to primary caregivers.

We aim to include $n=700$ adolescents and young adults aged 15-21 years in the trial. They will be randomly assigned to either receive the online CDP or online psychoeducation only. Participants in both groups will be assessed at the beginning (baseline; T0), 4 (post-line; T1), 12 , and 18 months after the initial baseline assessment (follow-up; T2, T3) in order to evaluate treatment effects. The flowchart of the trial is shown in Fig. 1. The schedule of this trial is shown in Fig. 2 (Additional file 1).

Recruitment is conducted online through social media (such as Instagram and Facebook), websites, and online forums. Furthermore, flyers will be printed and disseminated at the clinical centers participating in the study (Berlin/Neuruppin, Heidelberg, Landau, Mannheim, Ulm). All eligible participants registering on the central project website will be offered to participate in the online intervention.

\section{Inclusion and exclusion criteria}

Based on the criterion A of the NSSI disorder provided in the Diagnostic and Statistical Manual of Mental Disorders (DSM), fifth edition, section three [3], eligible participants are required to have engaged in NSSI on at least five days during the last 12 months. Eligible participants are aged 15-21 years. Furthermore, all participants need to provide informed consent online by clicking a checkbox. Participants will be excluded if they are currently receiving individual psychotherapy (inpatient and outpatient).

\section{Procedure and randomization}

Before inclusion, participants will be provided with written information on the study's background, aims

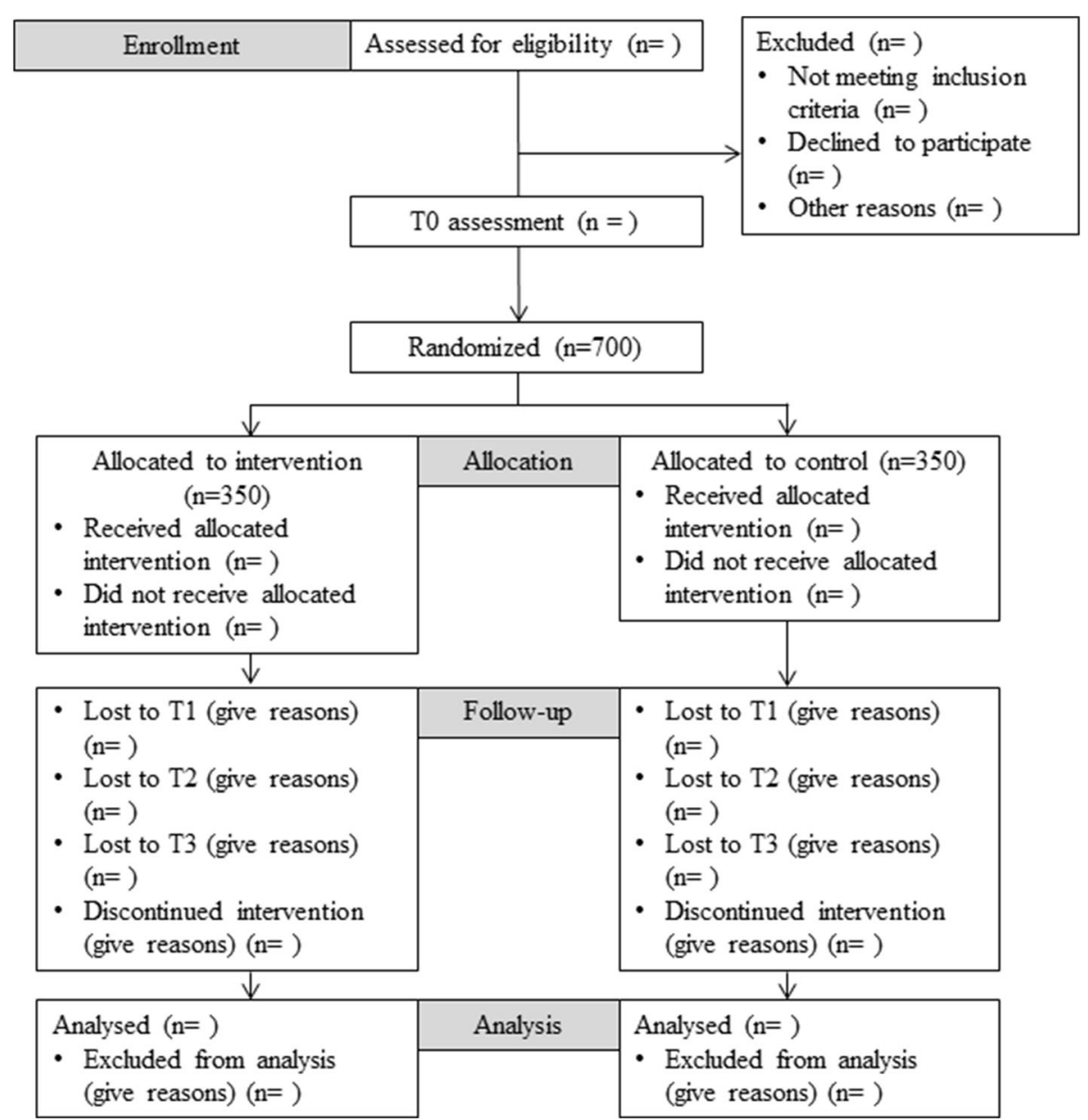

Fig. 1 Consolidated Standards of Reporting Trials (CONSORT) 2010 flow diagram 


\begin{tabular}{|c|c|c|c|c|c|c|c|}
\hline \multirow[b]{3}{*}{ TIMEPOINT** } & \multicolumn{7}{|c|}{ STUDY PERIOD } \\
\hline & \multirow{2}{*}{$\begin{array}{c}\text { Enrolment } \\
\begin{array}{c}\boldsymbol{t}_{0} \\
\text { (baseline) }\end{array} \\
\end{array}$} & \multirow{2}{*}{$\begin{array}{c}\text { Allocation } \\
\begin{array}{c}\mathrm{t}_{0} \\
\text { (baseline) }\end{array} \\
\end{array}$} & \multicolumn{2}{|c|}{ Post-allocation } & \multicolumn{3}{|c|}{ Follow-up } \\
\hline & & & $\begin{array}{c}\text { After } \\
\text { randomizati } \\
\text { on }\end{array}$ & $\begin{array}{l}4 \text { months after } \\
\text { randomization }\end{array}$ & $\begin{array}{c}\boldsymbol{t}_{1} \\
\text { (after } 4 \text { months) }\end{array}$ & $\begin{array}{c}t_{2} \\
\text { (after } 12 \text { months) }\end{array}$ & $\begin{array}{c}t_{3} \\
\text { (after } 18 \text { months) }\end{array}$ \\
\hline \multicolumn{8}{|l|}{ ENROLMENT: } \\
\hline Eligibility screen & $\mathrm{x}$ & & & & & & \\
\hline Informed consent & $\mathrm{X}$ & & & & & & \\
\hline Allocation & & $\mathrm{x}$ & & & & & \\
\hline \multicolumn{8}{|l|}{ INTERVENTIONS: } \\
\hline \multicolumn{8}{|l|}{ CDP online } \\
\hline \multicolumn{8}{|l|}{ Psychoeducation } \\
\hline \multicolumn{8}{|l|}{ ASSESSMENTS: } \\
\hline NSSV SG & $\mathrm{X}$ & & & & $\mathrm{X}$ & $\mathrm{X}$ & $\mathrm{x}$ \\
\hline PSS & $\mathrm{x}$ & & & & $\mathrm{X}$ & $\mathrm{x}$ & $\mathrm{X}$ \\
\hline PHQ-A & $\mathrm{X}$ & & & & $x$ & $\mathrm{x}$ & $\mathrm{X}$ \\
\hline$S D Q$ & $\mathrm{x}$ & & & & $x$ & $\mathrm{x}$ & $x$ \\
\hline KIDSCREEN & $\mathrm{x}$ & & & & $\mathrm{x}$ & $x$ & $\mathrm{X}$ \\
\hline SAPAS & $\mathrm{x}$ & & & & $\mathrm{x}$ & $\mathrm{X}$ & $\mathrm{X}$ \\
\hline$B S L-23$ & $\mathrm{x}$ & & & & $\mathrm{X}$ & $\mathrm{X}$ & $\mathrm{X}$ \\
\hline
\end{tabular}

Fig. 2 SPIRIT figure: Schedule of enrollment, interventions, and assessments

and procedures, the online CDP and online psychoeducation, randomization, the advantages and risks of participating, data collection and data safety regulations, as well as voluntary participation.

Informed consent will be obtained after an initial screening phase within T0 to check for eligibility and before registering on the study website. Participants confirm by ticking a box on the website that they have read and understood the provided information and that they are willing to participate.

Within the self-report assessment and on the study platform, participants will be informed that there is no immediate check of the data they enter and thus no immediate measures can be guaranteed in case a participant reports acute suicidality. Participants will be made aware of emergency numbers in case of acute suicidality.

After providing informed consent, participants have to provide an email address as well as a username in order to register for the study. An activation link will be sent to the email address provided. Once the address has been verified, a unique code will be generated and assigned to the participant, in order to enable the matching of follow-up assessments.
After consent and registration to the platform, all eligible participants will be asked to complete a 45-min assessment within T0. After completion of the assessment at $\mathrm{T} 0$, participants will be randomized to the online CDP or the online psychoeducation and will be invited to participate in assessments at T1, T2, and T3. To promote participant retention, participants will be reminded to participate in the assessments via standardized e-mails at each point in time. Within a first e-mail, participants receive an invitation as well as a link to the assessment. After 3 and 14 days, participants receive reminder e-mails for the assessment. This applies to every assessment point.

To prevent bias, participants will be randomly assigned to receive either the online psychoeducation or the online CDP using block randomization (50/50). Participants will be randomized based on their study ID. Enrolment, generation of allocation sequence, and assignment of participants take place online and are therefore computer-based. Participants will be informed about their group affiliation by automatic message that pops up immediately after randomization. Blinding is not completely possible. Participants cannot be blinded due to the different nature of the interventions. Blinding 
of the researchers is not possible, because participants in the online CDP group receive case-management and personal chats as well as telephone calls.

\section{Data assessment}

All assessments will be conducted online. Thus, selfreport questionnaires will be used in this trial. The assessment at T0 takes place before randomization and the subsequent start of treatment. All assessment measures are conducted at every time point (T0, T1, T2, T3), except the evaluation of the online CDP, which only takes place at T1. Within a first screening phase of T0, participants are checked for eligibility. All eligible participants are invited to participate in the trial. Those not eligible for the trial because of current individual psychotherapy and/or too low frequency of NSSI are invited to participate in a separate non-interventional observational study.

\section{Assessment measures}

T0 comprises sociodemographic questions, which will gather information about gender, nationality, state of adolescents' and parents' education, and current living situation.

For detailed assessment of NSSI severity, we use the NSSV-SG [33], assessing the frequency and nature of NSSI within the past three months using 60 items. The evaluation of the psychometric properties in a clinical and an online sample of adolescents and young adults indicates high internal consistency (Cronbach $\alpha=0.73$ ), a test-retest reliability of $0.77 \quad(n=29$; interval: six weeks), and supports the validity of the questionnaire [34]. The KIDSCREEN-10 will be used to assess general health-related quality of life (HRQoL) in participants [35]. It consists of 10 items and was developed across Europe for use in children aged 8-18 years and is a valid instrument with acceptable retest reliability [35]. Internal consistency was high (Cronbach $\alpha=0.81$ ) [36]. To assess emotional and behavioral difficulties in children and adolescents, the Strengths and Difficulties Questionnaire (SDQ) [37] with 25 items will be used. A study, evaluating the validity of the German version of the SDQ, indicates that the SDQ is valid for most clinical and research purposes [38]. In addition, the questionnaire shows good internal consistency (Cronbach $\alpha=0.81$ ) [39]. The Borderline Symptom List-23 (BSL-23) [40] is a quantitative assessment of borderline-specific symptoms based on DSM-IV criteria [41]. It consists of 23 items and shows high internal consistency (Cronbach $\alpha=0.94$ ) [40]. The BSL-23 is a reliable and valid instrument to assess borderline-specific symptoms [40]. To assess personality disorders, the Self-report Standardised Assessment of Personality - abbreviated scale (SAPAS-SF) consisting of eight items [42] will be used. The SAPAS is a valid and reliable instrument to screen for personality disorders
[42, 43]. The internal consistency coefficients were rather low, in the range of 0.35-0.51 [44]. The Paykel Suicide Scale (PSS) [45] assesses the intensity of suicidal behaviors by level of intent and consists of five items. The Patient Health Questionnaire-9 for Adolescents (PHQ-A) [46] is a modified version of the Patient Health Questionnaire-9 (PHQ-9) [47]. The PHQ-9 measures depression severity and shows good internal consistency (Cronbach $\alpha=0.84$ ) [48]. The PHQ-A consists of nine items based on DSM-IV [41]. The PHQ-A is a reliable and valid measure of depression severity [46] and shows good.

Beyond that, additional items will be presented to evaluate the online CDP. Within 20 items, participants will rate how helpful the different sessions have been and what they have learned on a 5- and 6-point Likert Scale, respectively. Furthermore, the revised short form of the Working Alliance Inventory (WAI-SR) [49] consisting of 12 items was used to assess the therapeutic relationship. Internal consistency was excellent (Cronbach $\alpha>0.90$ ) [50]. Finally, a modified version of The Fragebogen zur Patientenzufriedenheit (ZUF-8; Patient Satisfaction Questionnaire) was used to assess treatment satisfaction. It consists of eight statements, which are answered on a 4-point scale ranging from 'very satisfied' to 'quite dissatisfied' [51].

\section{Participants' incentive}

Participants receive no direct financial compensation for participating in the study. However, they are informed about the chance to win a gift voucher $(€ 50)$ for their participation. After completion of $\mathrm{T} 1$ assessment, participants have the chance to win the voucher.

\section{Interventions}

$N=700$ participants will be randomized to one of the two possible treatment conditions (online CDP versus online psychoeducation). Table 1 provides an overview of the interventions in both groups. The platform is available for fourmonths for each participant after randomization. After a successful evaluation of the online CDP, the intervention could be disseminated to provide all individuals concerned with access to the online CDP. However, there are no plans concerning an immediate access to the intervention for the control group after conclusion of the trial.

\section{Online psychoeducation}

The online psychoeducation provides static psychoeducative content on the causes, consequences, and concomitants of NSSI. This module is available for both groups. Recommendations concerning the intensity of use are rather individual depending on the already existing knowledge on NSSI. Thus, the intensity of use 
Table 1 Overview of interventions

\begin{tabular}{|c|c|}
\hline Group affiliation & Intervention \\
\hline \multirow[t]{5}{*}{ Both groups } & Online psychoeducation: \\
\hline & $\begin{array}{l}\text { - basic facts on NSSI, NSSI and emotions, NSSI } \\
\text { and development, treatment for NSSI in terms } \\
\text { of plain information }\end{array}$ \\
\hline & - static content \\
\hline & $\begin{array}{l}\text { - dose of the psychoeducative intervention } \\
\text { is determined by the user }\end{array}$ \\
\hline & - access for four months \\
\hline Control group & Online psychoeducation \\
\hline \multirow[t]{5}{*}{ Intervention group } & Online psychoeducation \\
\hline & Monitoring module: \\
\hline & $\begin{array}{l}\text { - weekly assessments on NSSI and the use } \\
\text { of skills for individual feedback }\end{array}$ \\
\hline & Add-on online CDP: \\
\hline & $\begin{array}{l}\text { - access to } 10 \text { different sessions: (1) identifying } \\
\text { resons for NSSI, getting to know skills; } \\
\text { (2) promoting motivation; (3) getting to } \\
\text { know and dealing with feelings; } \\
\text { (4) establishing positive activities; } \\
\text { (5) understanding the links between } \\
\text { thoughts, feelings, and behaviors and } \\
\text { learning how to find a more helpful way } \\
\text { of thinking; (6) identifying core beliefs and } \\
\text { rules of living; (7) identifying coping } \\
\text { strategies; (8) promoting assertiveness; } \\
\text { (9) identifying strategies and skills that are } \\
\text { based on the concepts of mindfulness and } \\
\text { distress tolerance; (10) exploring triggers } \\
\text { for NSSI, reviewing coping strategies } \\
\text { - techniques: chat and telephone calls } \\
\text { with case managers, moderated group } \\
\text { chat, exercises, videos, quizzes } \\
\text { - access for four months }\end{array}$ \\
\hline
\end{tabular}

is determined by the participant and tracked as a variable of interest.

\section{Online CDP}

The online CDP group is offered an add-on online intervention. The face-to-face, short-term intervention is based on elements of CBT and DBT and is specifically tailored for the treatment of NSSI in adolescents. The treatment length is $8-12$ sessions. The treatment consists of four modules that can be expanded by optional exercises. Module 1 focuses on providing knowledge about CBT and NSSI as well as promoting therapy motivation. The focus of module 2 is on identifying the reasons for the NSSI. In module 3 , the patients are encouraged to test alternative behaviors to NSSI and module 4 comprises the stabilization of the alternative behaviors. The content of the intervention is structured in a manual for participants and a separate manual for therapists that is feasible for translation into an online intervention. The modules in the manual have all been developed based on a comprehensive literature review on NSSI including associated psychological phenomena. Within the online CDP, the intervention is delivered via personal chat or telephone calls with the case managers and assisted by automatic content of the web-based platform, which provides 10 different sessions. The sessions comprise content of the face-to-face manual adapted for online use. In addition to text, participants are provided with exercises and quizzes to deepen their theoretical knowledge on the one hand and to ensure understanding of the provided content on the other hand. In addition, videos of two fictitious patients are provided in every session. These fictitious patients present common problems and how they deal with them. The online CDP group will further have access to a moderated online group chat facilitating exchange with other participants and providing peer support. Additionally, all participants in the online CDP group are monitored for NSSI and the use of skills on a weekly basis to receive an individual feedback on their progress. The intervention will solely be provided over the Internet.

Participants are encouraged to use the online CDP as often as they want to. There is no restriction or specification concerning the intensity of use. Again, the use of the online CDP is determined by the individual. Individual contacts with the case managers via personal chat or telephone call are regularly offered once a week. However, further appointments are possible if particular circumstances require additional support. Group chats also take place once a week.

All participants are permitted to seek treatment outside of the clinical trial if necessary. The nature and frequency/dose of interventions outside of the trial will be assessed using structured questionnaires.

\section{Emergency procedure}

If participants within the online CDP group indicate serious suicide thoughts within a chat or telephone call with a case manager, suicidality is clarified by the respective case manager in a first step, as well as by the responsible psychiatrist, as needed. Participants will then be referred to emergency numbers.

There are no general discontinuation criteria for the trial.

\section{Staff}

All case managers are clinical psychologists who receive a priori training in the online CDP intervention. Furthermore, case managers will receive weekly supervision by a specifically trained clinical psychologist with a clinical background in CBT and DBT to ensure the quality of treatment. In addition, monthly external supervision will be offered by a specifically trained psychotherapist in CBT and DBT. Beyond that, psychiatrists both for children and adolescents as well as for adults who also receive a priori 
training in the online $\mathrm{CDP}$ are available in cases of emergency. All case managers are based in Heidelberg.

\section{Sample size}

Power analysis was based on reported effect sizes of CBT in the reduction of NSSI, drawing on the most extensive meta-analysis in the field [52] reporting a main effect for the reduction of NSSI in $n=8 / 14$ studies; $g=-0.27,95 \%$ CI $[-0.17,0.38], \mathrm{z}=4.96, p=0.001)$. Most studies included in the analysis used a treatment as usual (TAU) control group design. Comparing online CDP against an online psychoeducation we expect similar effects. Power analysis was calculated for F-statistics using an alpha-error probability of 0.05 . The estimation model was based on a mixed effect linear regression with main effects (repeated measures and group) and their interaction. We considered covariates with potential impact on the outcome (sex, age). Based on the calculation, a minimum sample size of $n=344$ is necessary to reveal significant effects at a critical $F$-value of $F=1.859$. Based on this estimation, the study would allow for up to $51 \%$ drop-out and still be sufficiently powered revealing effects in a completer analysis.

\section{Statistical analysis}

The primary hypothesis will be addressed using a mixed linear regression analysis with robust variance estimation, assessing the frequency of NSSI within the past 3 months at $\mathrm{T} 0$ and $\mathrm{T} 2$. We will consider main and interaction effects of the two main effects: (1) group allocation; and (2) time of assessment within repeated measures. The individuals' ID will be used as random effect variable. In secondary analyses, potential sociodemographic and clinical mediators and predictors of intervention response will be investigated. Missing data and individuals withdrawn from the trial will be handled using an intentionto-treat (ITT) approach. All participants randomized will be considered in the analyses. In the case of drop-outs or missing data, we will use a last-value-carried-forward imputation method, assuming that those who dropped out of the intervention showed no improvement in NSSI relative to baseline.

\section{Data safety}

The confidentiality of research participants is secured by providing unique study identifiers unrelated to the real name.

The Coordination Centre for Clinical Trials (KKS) in Heidelberg is involved in preparing the investigator site file. Due to the online-based nature of the intervention, classical site visits are not necessary. Instead, pre-trial visits in terms of profound explanation of online content and all aspects related to delivery of the intervention took place. In addition, an independent data safety and monitoring board (DSMB) was established to monitor the collection and analyses of data.

Computerized assessments guarantee the highest level of data integrity and quality, i.e. missing data will be minimized and false data entry will be prevented. Online access allows for continuous monitoring of data collection, documentation of access logs, and traceability of all entered data (user and timestamp) as well as restoration of all previous states. A Distributed Replicated Block Device (DRBD)-based cluster will provide synchronous replication of all data during data entry on two separate servers and highest availability. In addition, full and incremental backups will be conducted following a predefined back-up plan. Data storage and transfer will be encrypted. Access to the data will be strictly limited to authorized persons and will be passwordprotected. All servers are located at the University Hospital Heidelberg.

\section{Harms}

In view of the non-invasive intervention, the risk for participants is considered marginal. Irrespective of the individual allocation to one of the treatment arms, participating adolescents and young adults benefit from receiving psychoeducation on NSSI and associated mental distress. Adolescents will be instructed on the crisis procedures mentioned above. There is no obvious risk for participants. The independent KSS Heidelberg will provide expert advice and monitoring regarding all aspects of safety. Beyond that, the DSMB will be informed about adverse events, such as suicidal acts.

\section{Ethical issues and dissemination}

The study will be conducted in accordance to the declaration of Helsinki and the rules for physicians of the medical association ("Landesärztekammer") of Baden-Württemberg in their currently valid version. Study participation is voluntary. Consent can be withdrawn at any time without stating the reason and without any individual disadvantage for subsequent medical care. All participants will need to consent electronically to the terms of the study. In case of study withdrawal, previously collected data will be destroyed if desired unless data are already included in analyses. A waiver for parental consent was obtained from the institutional review board of the medical faculty at the University of Heidelberg. Ethics committees are able to waive paternal informed consent if studies are expected to result in great benefits for a specific target group or if studies could not be conducted otherwise [53]. Both points apply to the present study. Beyond that, the study is a noninvasive low-risk study and within the medical community, the age of 14 is considered to be the age when minor participants are able to consent to participation in low-risk studies $[54,55]$. Cognitive abilities are comparable to those 
in adults at the age of 14 years [56]. Furthermore, the study is about reducing obstacles in receiving professional help. Thus, asking for parental informed consent would increase hurdles, especially for adolescents who are often struggling to receive adequate professional help [57].

The study protocol has been approved by the ethics committee in Heidelberg for consulting of professional conduct before the start of our study.

In the case of relevant protocol modifications, the institutional review board of the medical faculty at the University of Heidelberg will be informed and an amendment will be submitted. Furthermore, information within the German Clinical Trials Register will be updated to inform the public about possible changes.

All confidential information is subject to medical confidentiality and to the requirements of the European, Federal and State Data Protection Act (Europäische Datenschutzgrundverordnung, EU-DSGVO, Bundesdatenschutzgesetz, BDSG and Landesdatenschutzgesetz, LDSG). The data will be stored and processed in a pseudonymized manner. No third parties will gain insights into the original data.

Beyond regular journal publication, general strategies are planned to disseminate trial results: coordination and participation in scientific meetings (scientific dissemination) as well as dissemination of materials created in the subproject STAR-TRAIN, which develops practical instructions for the contact to adolescents and young adults with NSSI in primary care practice (dissemination to the public). The use of professional writers is not intended. Access to the protocol is ensured through the registration and regular update of the trial in the German Clinical Trials Register (STAR: Self-Injury: Treatment, Assessment, Recovery - Online Intervention for Adolescent Nonsuicidal Self-Injury - A randomized controlled trial; http://www.drks.de; DRKS00014623; registration date: 22 May 2018).

\section{Discussion}

NSSI is a significant problem in adolescents and young adults, associated with severe psychopathological distress and potential long-term consequences [4-7]. Available intervention options are limited in their outreach and often fail to reach youth in rural areas and those with low help-seeking behavior. An online intervention may help to overcome existing barriers and improve general access to care for adolescents and young adults engaging in NSSI. However, so far, no study has addressed the effectiveness of online interventions for this target group. Thus, this is the first RCT to develop and evaluate an Internet-based short-term treatment for NSSI in adolescence and young adulthood. The present study and related intervention make several advances in the treatment of NSSI and build upon previous work in innovative ways.

First, we focus on NSSI, which is a significant issue in the mentioned age group. Second, we provide an evaluated short-term program addressing NSSI specifically. Third, we use an Internet-based approach to deliver therapy content. Intervening via this medium offers a number of advantages over face-to-face interventions and promises to reach a significant number of affected individuals regardless of their place of residence. In addition, help-seeking is considerably low in the target group and online interventions may lower barriers for young people to seek help.

\section{Limitations}

Despite the mentioned advantages, potential limitations of the study design should be acknowledged. Participant drop-out is a potential challenge, particularly given the online nature of the intervention. Based on a Cochrane Review [58], attrition rates from 4\% [59] up to 21\% [60] were reported within face-to-face treatments of adolescent self-harm (including NSSI). Concerning online interventions for adolescents with diagnoses of depression [61, 62] and anxiety [61, 63], drop-out rates from 6\% [64] up to $31 \%$ [65] were reported. Considering the mentioned studies, an attrition rate of $30 \%$ to $\mathrm{T} 1$ and $50 \%$ to $\mathrm{T} 2$ is expected, expecting $n=504$ adolescents to complete the intervention. The study is suitably powered even down to a completer sample of $n=344$ adolescents.

Another potential limitation is that all participants will be able to access alternative treatments outside of the trial. Information will be collected from participants about menthal health service usage and the potential impact of this on outcomes will be statistically controlled for.

In addition, we are not able to diagnose mental disorders because of the exclusive use of surveys. This is due to the online character of the study.

The provision of an online intervention, tailored to the needs of adolescents and young adults, may provide an easily accessible, cost-effective, and flexible medium for improving mental health outcomes for affected individuals.

\section{Trial status}

Protocol version 1.0, 25 April 2018.

The trial is scheduled to be completed by 31 October 2021. Recruitment began on 1 November 2018 and recruitment will be completed approximately by 31 October 2020 .

\section{Additional file}

Additional file 1: SPIRIT 2013 Checklist: Recommended items to address in a clinical trial protocol and related documents*. (DOC $123 \mathrm{~kb}$ ) 


\section{Abbreviations}

BDSG: Bundesdatenschutzgesetz; BPD: Borderline Personality Disorder; BSL23: Borderline Symptom List-23; CBT: Cognitive Behavioral Therapy; CDP: Cutting Down Programme; DBT-A: Dialectical Behavior Therapy for Adolescents; DRBD: Distributed Replicated Block Device; DSM-5: Diagnostic and Statistical Manual of Mental Disorders, Fifth edition; EU-

DSGVO: Europäische Datenschutzgrundverordnung; ITT: Intention-to-treat KIDSCREEN: Health-Related Quality of Life Questionnaire for Children and Young People; KKS: Coordination Centre for Clinical Trials;

LDSG: Landesdatenschutzgesetz; MACT: Manual-assisted Cognitive Behavior Therapy; MBT-A: Mentalization-Based Treatment for Adolescents; NSSI: Nonsuicidal self-injury; NSSV-SG: NSSI Severity Questionnaire; PHQ-A: Patient Health Questionnaire-9 for Adolescents; PSS: Paykel Suicide Scale; RCT: Randomized controlled trial; SAPAS-SF: Self-report Standardized Assessment of Personality - abbreviated scale; SDQ: Strengths and Difficulties Questionnaire; TAU: Treatment as usual

\section{Acknowledgements}

We thank the whole STAR team: Paul Plener, Rebecca Brown, Cedric Sachser, Daniela Harsch, Elisa Drews, Patrice van der Venne, Christian Schmahl, Birgit Abler, Andreas Chiocchetti, Inga Niedtfeld, Christine Margarete Freitag, Sarah Kuniss, Vera Münch, Maurizio Sicorello, Tina In-Albon, Romuald Brunner, Laura Kraus, Ulrich Ebner-Priemer, Philip Santangelo, Jörg Fegert, Michael Karenfort, Ulrike Hoffmann, Elisa König, Michael Kölch, Nicole Wiggert, Juliane Rausch.

\section{Authors' contributions}

MK is responsible for the design and coordination of the study. JK participated in the design and coordination of the study and helped to write the first draft of the manuscript. SB and MM are responsible for the technical part of the trial and revised the manuscript critically. GFW, MM, SCH, FR, RB, PLP, CS, CS, MK, and TIA revised the manuscript critically. AE participated in the design and the coordination of the study and wrote the first draft of the manuscript. All authors read and approved the final manuscript.

\section{Funding}

This trial is funded by the German Federal Ministry of Education and Research (BMBF; 01GL1747B). None of the project partners involved in the trial has a potential conflict of interest to declare.

\section{Availability of data and materials}

Not applicable.

\section{Ethics approval and consent to participate}

The institutional review board of the medical faculty at the University of Heidelberg approved the study (Ethics Committee No. S-288/2018). All participants will submit their informed consent before study inclusion. We obtained a waiver of parental consent from the institutional review board of the medical faculty at the University of Heidelberg in order to ensure feasibility.

\section{Consent for publication}

Not applicable.

\section{Competing interests}

PLP received a speaker's honorarium from Shire. He was involved in clinical studies for Lundbeck and Servier. The other authors declare that they have no competing interests.

\section{Author details}

University Hospital of Child and Adolescent Psychiatry and Psychotherapy, University of Bern, Bern, Switzerland. ${ }^{2}$ Section for Translational Psychobiology in Child and Adolescent Psychiatry, Department of Child and Adolescent Psychiatry, Centre for Psychosocial Medicine, University Hospital Heidelberg, Heidelberg, Germany. ${ }^{3}$ Centre for Psychotherapy Research, University Hospital Heidelberg, Heidelberg, Germany. ${ }^{4}$ Clinic for Child and Adolescent Psychiatry, Centre for Psychosocial Medicine, University Hospital Heidelberg Heidelberg, Germany. ${ }^{5}$ Department of General Psychiatry, Centre for Psychosocial Medicine, University Hospital Heidelberg, Heidelberg, Germany ${ }^{6}$ Department of Child and Adolescent Psychiatry and Psychotherapy, University Hospital Ulm, Ulm, Germany. ${ }^{7}$ Department of Clinical Child and Adolescent Psychology and Psychotherapy, Faculty of Psychology, University of Koblenz-Landau, Landau, Germany. ${ }^{8}$ Department for Child and Adolescent
Psychiatry and Psychotherapy, Medical School Brandenburg, Neuruppin, Germany. ${ }^{9}$ Department of Child and Adolescent Psychiatry, Medical University of Vienna, Vienna, Austria. ${ }^{10}$ Department of Psychosomatic Medicine and Psychotherapy, Central Institute of Mental Health, Medical Faculty Mannheim, Heidelberg University, Mannheim, Germany.

Received: 2 November 2018 Accepted: 5 June 2019

Published online: 12 July 2019

\section{References}

1. Swannell SV, Martin GE, Page A, Hasking P, St John NJ. Prevalence of nonsuicidal self-injury in nonclinical samples: systematic review, metaanalysis and meta-regression. Suicide Life Threat Behav. 2014:44:273-303.

2. Muehlenkamp JJ, Claes L, Havertape L, Plener PL. International prevalence of adolescent non-suicidal self-injury and deliberate self-harm. Child Adolesc Psychiatry Ment Health. 2012;6:10.

3. American Psychiatric Association. Diagnostic and Statistical Manual of Mental Disorders, Fifth Edition. Arlington: American Psychiatric Association; 2013.

4. Hawton K, Saunders KE, O'Connor RC. Self-harm and suicide in adolescents. Lancet. 2012;379:2373-82.

5. Asarnow JR, Porta G, Spirito A, Emslie G, Clarke G, Wagner KD, et al. Suicide attempts and nonsuicidal self-injury in the treatment of resistant depression in adolescents: findings from the TORDIA study. J Am Acad Child Adolesc Psychiatry. 2011;50:772-81.

6. Koenig J, Brunner R, Fischer-Waldschmidt G, Parzer P, Plener PL, Park J, et al. Prospective risk for suicidal thoughts and behaviour in adolescents with onset, maintenance or cessation of direct self-injurious behaviour. Eur Child Adolesc Psychiatry. 2017;26:345-54.

7. Brunner R, Kaess M, Parzer P, Fischer G, Carli G, Hoven C W, et al. Life-time prevalence and psychosocial correlates of adolescent direct self-injurious behavior: A comparative study of findings in 11 European countries. J Child Psychol Psychiatry. 2014:55:337-48.

8. In-Albon T, Ruf C, Schmid M. Proposed diagnostic criteria for the DSM-5 of nonsuicidal self-injury in female adolescents: diagnostic and clinical correlates. Psychiatry J. 2013;2013:1-12.

9. Plener PL, Schumacher TS, Munz LM, Groschwitz RC. The longitudinal course of non-suicidal self-injury and deliberate self-harm: a systematic review of the literature. Borderline Personal Disord Emot Dysregulation. 2015;2:2.

10. Bornovalova MA, Hicks BM, lacono WG, McGue M. Stability, change, and heritability of borderline personality disorder traits from adolescence to adulthood: A longitudinal twin study. Dev Psychopathol. 2009;21:1335-53.

11. Stepp SD, Whalen DJ, Scott LN, Zalewski M, Loeber R, Hipwell AE. Reciprocal effects of parenting and borderline personality disorder symptoms in adolescent girls. Dev Psychopathol. 2014;26:361-78.

12. Turner BJ, Austin SB, Chapman AL. Treating nonsuicidal self-injury: a systematic review of psychological and pharmacological interventions. Can J Psychiatr. 2014;59:576-85.

13. Andover MS, Schatten HT, Morris BW, Miller IW. Development of an intervention for nonsuicidal self-injury in young adults: an open pilot trial. Cogn Behav Pract. 2015;22:491-503.

14. Brausch AM, Girresch SK. A review of empirical treatment studies for adolescent nonsuicidal self-injury. J Cogn Psychother. 2012:26:3-18.

15. Mehlum L, Tørmoen AJ, Ramberg M, Haga E, Diep LM, Laberg S, et al. Dialectical behavior therapy for adolescents with repeated suicidal and selfharming behavior: a randomized trial. J Am Acad Child Adolesc Psychiatry. 2014;53:1082-91

16. Ougrin D, Tranah T, Stahl D, Moran P, Asarnow JR. Therapeutic interventions for suicide attempts and self-harm in adolescents: systematic review and meta-analysis. J Am Acad Child Adolesc Psychiatry. 2015;54:97-107.e2.

17. Rossouw TI, Fonagy P. Mentalization-based treatment for self-harm in adolescents: a randomized controlled trial. J Am Acad Child Adolesc Psychiatry. 2012;51:1304-13.

18. McMain SF, Guimond T, Barnhart R, Habinski L, Streiner DL. A randomized trial of brief dialectical behaviour therapy skills training in suicidal patients suffering from borderline disorder. Acta Psychiatr Scand. 2017:135:138-48.

19. Fischer G, Brunner R, Parzer P, Resch F, Kaess M. Short-term psychotherapeutic treatment in adolescents engaging in non-suicidal selfinjury: a randomized controlled trial. Trials. 2013;14:294. 
20. Cotter J, Kaess M, Yung AR. Childhood trauma and functional disability in psychosis, bipolar disorder and borderline personality disorder: a review of the literature. Ir J Psychol Med. 2015;32:21-30.

21. Doyle L, Treacy MP, Sheridan A. Self-harm in young people: Prevalence, associated factors, and help-seeking in school-going adolescents. Int J Ment Health Nurs. 2015;24:485-94.

22. Pumpa M, Martin $\mathrm{G}$. The impact of attitudes as a mediator between sense of autonomy and help-seeking intentions for self-injury. Child Adolesc Psychiatry Ment Health. 2015;9:27.

23. Hasking P, Rees CS, Martin G, Quigley J. What happens when you tell someone you self-injure? The effects of disclosing NSSI to adults and peers. BMC Public Health. 2015;15:1039.

24. Harris IM, Roberts LM. Exploring the use and effects of deliberate self-harm websites: an Internet-based study. J Med Internet Res. 2013;15:e285.

25. Lewis SP, Heath NL, Michal NJ, Duggan JM. Non-suicidal self-injury, youth, and the Internet: What mental health professionals need to know. Child Adolesc Psychiatry Ment Health. 2012;6:13.

26. Dyson MP, Hartling L, Shulhan J, Chisholm A, Milne A, Sundar P, et al. A systematic review of social media use to discuss and view deliberate selfharm acts. PLoS One. 2016;11:e0155813.

27. Ranney ML, Choo EK, Spirito A, Mello MJ. Adolescents' preference for technology-based emergency department behavioral interventions: does it depend on risky behaviors? Pediatr Emerg Care. 2013;29:475-81.

28. Younes N, Chollet A, Menard E, Melchior M. E-mental health care among young adults and help-seeking behaviors: a transversal study in a community sample. J Med Internet Res. 2015;17:e123.

29. Kaess M, Brunner R, Parzer P, Carli V, Apter A, Balazs JA, et al. Risk-behaviour screening for identifying adolescents with mental health problems in Europe. Eur Child Adolesc Psychiatry. 2014;23:611-20.

30. Evans K, Tyrer P, Catalan J, Schmidt U, Davidson K, Dent J, et al. Manualassisted cognitive-behaviour therapy (MACT): a randomized controlled trial of a brief intervention with bibliotherapy in the treatment of recurrent deliberate self-harm. Psychol Med. 1999;29:19-25.

31. Taylor LM, Oldershaw A, Richards C, Davidson K, Schmidt U, Simic M. Development and pilot evaluation of a manualized cognitivebehavioural treatment package for adolescent self-harm. Behav Cogn Psychother. 2011:39:619-25.

32. Renton $T$, Tang $H$, Ennis N, Cusimano MD, Bhalerao S, Schweizer TA, et al. Web-based intervention programs for depression: a scoping review and evaluation. J Med Internet Res. 2014;16:e209.

33. In-Albon T, Niedtfeld I, Kaess M. NSSI Severity Questionnaire (NSSV-SG). Landau: University of Koblenz-Landau; 2017.

34. Heitz C. Validation of the Severity Questionnaire of Nonsuicidal Self-Injury (NSSVSG) - Primary focus on adolescence. Landau: University of Koblenz-Landau; 2017.

35. Ravens-Sieberer U, Erhart M, Rajmil L, Herdman M, Auquier P, Bruil J, et al. Reliability, construct and criterion validity of the KIDSCREEN-10 score: a short measure for children and adolescents' well-being and health-related quality of life. Qual Life Res Int J Qual Life Asp Treat Care Rehabil. 2010;19:1487-500.

36. Erhart M, Ottova V, Gaspar T, Jericek H, Schnohr C, Alikasifoglu M, et al. Measuring mental health and well-being of school-children in 15 European countries using the KIDSCREEN-10 Index. Int J Public Health. 2009;54:160-6.

37. Goodman R. The Strengths and Difficulties Questionnaire: a research note. J Child Psychol Psychiatry. 1997;38:581-6.

38. Klasen H, Woerner W, Wolke D, Meyer R, Overmeyer S, Kaschnitz W, et al. Comparing the German versions of the strengths and difficulties questionnaire (SDQ-Deu) and the child behavior checklist. Eur Child Adolesc Psychiatry. 2000;9:271-6.

39. Goodman R. Psychometric properties of the Strengths and Difficulties Questionnaire. J Am Acad Child Adolesc Psychiatry. 2001;40:1337-45.

40. Bohus M, Kleindienst N, Limberger MF, Stieglitz R-D, Domsalla M, Chapman AL, et al. The short version of the Borderline Symptom List (BSL-23): development and initial data on psychometric properties. Psychopathology. 2009;42:32-9.

41. American Psychiatric Association. Diagnostic and statistical manual of mental disorders: DSM-IV. Washington, DC: American Psychiatric Association; 1994.

42. Moran P, Leese M, Lee T, Walters P, Thornicroft G, Mann A. Standardised Assessment of Personality - Abbreviated Scale (SAPAS): preliminary validation of a brief screen for personality disorder. Br J Psychiatry J Ment Sci. 2003;183:228-32

43. Fok ML-Y, Seegobin S, Frissa S, Hatch SL, Hotopf M, Hayes RD, et al. Validation of the standardised assessment of personality--abbreviated scale in a general population sample. Personal Ment Health. 2015;9:250-7.
44. Germans S. A search for personality disorder screenings tools: A helping hand in the daily practice for the busy clinician. Universiteit van Tilburg; 2011.

45. Paykel ES, Myers JK, Lindenthal JJ, Tanner J. Suicidal feelings in the general population: a prevalence study. Br J Psychiatry J Ment Sci. 1974;124:460-9.

46. Johnson JG, Harris ES, Spitzer RL, Williams JBW. The patient health questionnaire for adolescents: validation of an instrument for the assessment of mental disorders among adolescent primary care patients. J Adolesc Health Off Publ Soc Adolesc Med. 2002;30:196-204.

47. Kroenke K, Spitzer RL, Williams JB. The PHQ-9: validity of a brief depression severity measure. J Gen Intern Med. 2001;16:606-13.

48. Ganguly S, Samanta M, Roy P, Chatterjee S, Kaplan DW, Basu B. Patient Health Questionnaire-9 as an effective tool for screening of depression among Indian adolescents. J Adolesc Health. 2013;52:546-51.

49. Wilmers F, Munder T, Leonhart R, Herzog T, Plassmann R, Barth J, et al. Die deutschsprachige Version des Working Alliance Inventory-short revised (WAI-SR)-Ein schulenübergreifendes, ökonomisches und empirisch validiertes Instrument zur Erfassung der therapeutischen Allianz. Klin Diagn Eval. 2008;1:343-58.

50. Munder T, Wilmers F, Leonhart R, Linster HW, Barth J. Working Alliance Inventory-Short Revised (WAI-SR): psychometric properties in outpatients and inpatients. Clin Psychol Psychother. 2009;17(3):231-9.

51. Schmidt J, Lamprecht F, Wittmann WW. Zufriedenheit mit der stationären Versorgung. Entwicklung eines Fragebogens und erste Validitätsuntersuchungen. PPmP Psychother Psychosom Med Psychol. 1989;39(7):248-55.

52. Labelle R, Pouliot $L$, Janelle A. A systematic review and meta-analysis of cognitive behavioural treatments for suicidal and self-harm behaviours in adolescents. Can Psychol Can. 2015;56:368.

53. Health UD of, Services H. Code of Federal Regulations Title 45 Public Welfare Part 46 Protection of Human Subjects. Wash DC: US Dept Health Hum Serv; 2009.

54. Schachter D, Kleinman I, Harvey W. Informed consent and adolescents. Can J Psychiatr. 2005;50:534-40.

55. Levine RJ. Adolescents as research subjects without permission of their parents or guardians: Ethical considerations. J Adolesc Health. 1995;17:287-97.

56. Santelli JS, Rosenfeld WD, RH DR, Dubler N, Morreale M, English A, et al. Guidelines for adolescent health research: a position paper of the Society for Adolescent Medicine. J Adolesc Health. 1995;17:270-6.

57. Gulliver A, Griffiths KM, Christensen H. Perceived barriers and facilitators to mental health help-seeking in young people: a systematic review. BMC Psychiatry. 2010;10:113.

58. Hawton K, Witt KG, Taylor Salisbury TL, Arensman E, Gunnell D, Townsend E, et al. Interventions for self-harm in children and adolescents. Cochrane Database Syst Rev. 2015;12:CD012013.

59. Harrington R, Kerfoot M, Dyer E, McNiven F, Gill J, Harrington V, et al. Randomized trial of a home-based family intervention for children who have deliberately poisoned themselves. J Am Acad Child Adolesc Psychiatry. 1998;37:512-8.

60. Cotgrove A, Zirinsky L, Black D, Weston D. Secondary prevention of attempted suicide in adolescence. J Adolesc. 1995;18:569-77.

61. Ebert DD, Zarski A-C, Christensen H, Stikkelbroek Y, Cuijpers $P$, Berking $M$, et al. Internet and computer-based cognitive behavioral therapy for anxiety and depression in youth: a meta-analysis of randomized controlled outcome trials. PLoS One. 2015;10:e0119895.

62. Zhou X, Hetrick SE, Cuijpers P, Qin B, Barth J, Whittington CJ, et al. Comparative efficacy and acceptability of psychotherapies for depression in children and adolescents: A systematic review and network meta-analysis. World Psychiatry. 2015;14:207-22

63. Podina IR, Mogoase C, David D, Szentagotai A, Dobrean A. A meta-analysis on the efficacy of technology mediated CBT for anxious children and adolescents. J Ration-Emotive Cogn-Behav Ther. 2016;34:31-50.

64. Stasiak K, Hatcher S, Frampton C, Merry SN. A pilot double blind randomized placebo controlled trial of a prototype computer-based cognitive behavioural therapy program for adolescents with symptoms of depression. Behav Cogn Psychother. 2014;42:385-401.

65. Fleming T, Dixon R, Frampton C, Merry S. A pragmatic randomized controlled trial of computerized CBT (SPARX) for symptoms of depression among adolescents excluded from mainstream education. Behav Cogn Psychother. 2012:40:529-41.

\section{Publisher's Note}

Springer Nature remains neutral with regard to jurisdictional claims in published maps and institutional affiliations. 